\title{
Influência da pré-cloração na clarificação de águas para abastecimento utilizando coagulante à base de tanino
}

\author{
Pre-chlorination effects on drinking water treatment using tannin-based coagulant
}

\author{
Jéssica Rodrigues Silveira'* ${ }^{\circledR}$, Valéria Menezes de Souza' ${ }^{\circledR}$, Renata Medici Frayne Cuba' ${ }^{\oplus}$, \\ Francisco Javier Cuba Teran' ${ }^{10}$
}

\begin{abstract}
RESUMO
Diferentes técnicas de tratamento de água destinada ao abastecimento público, como a pré-cloração e o uso de coagulantes orgânicos, têm sido relatadas como alternativas econômica, técnica e ambientalmente viáveis. Entretanto, o resultado da interação entre esses processos deve ser estudado para sua aplicação com segurança. Dessa forma, este estudo objetivou avaliar a influência da pré-cloração na clarificação de água para abastecimento público, utilizando coagulante à base de tanino. Os ensaios foram conduzidos em jar test com o uso de Tanfloc SG como coagulante, variando as dosagens de 2,5 a 45,0 mg L'-1, e de oxidante hipoclorito de sódio $(\mathrm{NaClO})$ nas concentrações de cloro residual livre $\left(\mathrm{HClO}_{(\mathrm{Naco})}\right)$ de 0,8 e 5,0 mg L'-1, no tratamento da água do ribeirão João Leite. As dosagens ótimas de coagulante foram determinadas para as seguintes condições de tratamento: adição somente de tanino; tanino e NaClO aplicados simultaneamente; e tanino adicionado após 30 min da précloração. Nessas condições, o pH de coagulação foi analisado de 6,0 a 8,5. Foram avaliados parâmetros como turbidez, cor aparente, $\mathrm{pH}$ e $\mathrm{HClO}_{\text {(Naclo) }}$. A melhor condição de tratamento com pré-cloração foi a adição simultânea de $\mathrm{NaClO}$ e coagulante tanino nas dosagens de $0,8 \mathrm{mg} \mathrm{L}^{-1}\left(\mathrm{HClO}_{(\mathrm{NaClO})}\right)$ e 10,0 mg $L^{1-1}$, respectivamente, com remoção de turbidez de $71,6 \%$ e cor aparente de $62,7 \%$. Os resultados demonstraram que a pré-cloração afetou negativamente o processo de tratamento, e para maior concentração de $\mathrm{HClO}_{(\mathrm{NaClO})}$ maior foi a dosagem de tanino requerida, sem apresentar aumento significativo nas eficiências de remoção de turbidez e cor aparente.
\end{abstract}

Palavras-chave: oxidação; hipoclorito de sódio; coagulação; tanino.

\begin{abstract}
Different techniques in the treatment of water intended for public supply such as pre-chlorination and use of organic coagulants have been reported as economic, technical and environmental viable alternatives. However, the result of the interaction between these processes should be studied for their safe application. Thus, this study aimed to evaluate the influence of prechlorination on coagulation and flocculation of water for public supply using a tannin-based coagulant. The assays were conducted in Jar Test using Tanfloc SG as a coagulant, varying the dosages from 2.5 to $45.0 \mathrm{mg} \mathrm{L}^{-1}$, and sodium hypochlorite oxidant $(\mathrm{NaClO})$ in the concentrations of free residual chlorine $\left(\mathrm{HClO}_{(\mathrm{NaClO})}\right)$ of 0.8 and $5.0 \mathrm{mg} \mathrm{L}^{-1}$, in the water treatment of João Leite River. Optimal coagulant dosages were determined for the following treatment conditions: addition of tannin only; tannin and $\mathrm{NaClO}$ applied simultaneously; and, tannin added after 30 min of pre-chlorination. Under these conditions, the coagulation $\mathrm{pH}$ was analyzed from 6.0 to 8.5. Parameters such as turbidity, apparent color, $\mathrm{pH}$ and $\mathrm{HClO}(\mathrm{NaClO})$ were evaluated. The best treatment condition with pre-chlorination was the simultaneous addition of $\mathrm{NaClO}$ and coagulant at dosages of $0.8 \mathrm{mg} \mathrm{L}^{-1}\left(\mathrm{HClO}_{(\mathrm{NaClO})}\right)$ and $10.0 \mathrm{mg} \mathrm{L}^{-1}$ respectively, with turbidity removal of $71.6 \%$ and apparent color of $62.7 \%$. In general, the results showed that pre-chlorination negatively affected the process being that the higher concentration of $\mathrm{HClO}_{(\mathrm{NaClO})}$ the higher tannin dosage, without presenting a significant increase in the efficiencies of turbidity and apparent color removal.
\end{abstract}

Keywords: oxidation; sodium hypochlorite; coagulation; tannin.

\section{INTRODUÇÃO}

Para atender aos padrões de potabilidade da Portaria de Consolidação n ${ }^{\circ} 5$ (BRASIL, 2017), as águas superficiais destinadas ao consumo humano são submetidas a diferentes etapas de tratamento, como pré-oxidação, coagulação,

floculação, decantação, filtração, fluoretação e desinfecção. Entre elas, as etapas de pré-oxidação e coagulação são avaliadas neste trabalho.

A pré-oxidação da água bruta tem sido utilizada em estações de tratamento de água (ETA) como técnica alternativa tanto para minimizar os problemas causados

口-

'Universidade Federal de Goiás - Goiânia (GO), Brasil.

Conflitos de interesse: os autores declaram não haver conflitos de interesse.

Financiamento: Coordenação de Aperfeiçoamento de Pessoal de Nível Superior (CAPES) - processos 88882.386574/2019-01 e 88882.385992/2019-01

*Autor correspondente: engjessicarodriguessilveira@gmail.com

Recebido: 30/03/2020 - Aceito: 13/04/2021 - Reg. ABES: 20200391 
por florações de cianobactérias (NETO \& NEYCOMBE, 2017) quanto para oxidar contaminantes orgânicos (substâncias húmicas) e inorgânicos (metais) que possam interferir nas demais etapas de tratamento (HELLER \& PÁDUA, 2006).

Adicionalmente, segundo Neto e Neycombe (2017), estudos têm demonstrado que a pré-oxidação da água pode ter efeito positivo sobre os processos convencionais de tratamento, por exemplo, reduzindo a dosagem de coagulante e melhorando a qualidade da água tratada. Entretanto, a maioria das pesquisas têm como base o uso de coagulantes inorgânicos, como sais de alumínio e ferro, cuja substituição por coagulantes orgânicos tem sido considerada nos últimos tempos em razão, principalmente, da elevada produção de volume de lodo não biodegradável, da alteração do $\mathrm{pH}$ da água, dos custos elevados de reagentes químicos e da possibilidade de formação de subprodutos tóxicos que prejudicam tanto a saúde humana quanto o ambiente (OLADOJA et al., 2017).

Entre os coagulantes orgânicos disponíveis no mercado com capacidade de atender às necessidades do tratamento de água, têm-se os polímeros catiônicos preparados com produtos naturais, como o tanino extraído da Acacia mearnsii (Acácia negra) (MANGRICH et al., 2014). A cationização de taninos é um procedimento químico que confere caráter catiônico à matriz orgânica do tanino de modo que as principais características, como solubilidade, estabilidade em diferentes intervalos de pH e atividade quelante de metais pesados são mantidas, enquanto outras, como o caráter positivo da molécula, necessário para a desestabilização de partículas coloidais aniônicas, são potencializadas (BELTRÁN-HEREDIA et al., 2010).

Dessa forma, estudos têm sido realizados para avaliar a eficiência de compostos à base de tanino como coagulante para a clarificação de águas e, em alguns casos, eles apresentaram melhores eficiências de tratamento quando comparados aos compostos inorgânicos (SANTOS et al., 2018).

Thakur e Choubey (2014) observaram remoção de 91,0\% de turbidez e de $57,3 \%$ de sólidos dissolvidos ao tratar amostra de água superficial com dosagem de $3,0 \mathrm{~mL} \mathrm{~L}^{-1}$ de extrato tanino $\left(0,01 \mathrm{~g} \mathrm{~mL}^{-1}\right)$ obtido da Acacia catechu. Skoronski et al. (2014) trataram água do Rio Tubarão (SC) com a concentração de $2,5 \mathrm{mg}$ $\mathrm{L}^{-1}$ de tanino (Tanfloc SG, Tanac S.A) e obtiveram resultados dos parâmetros cor e turbidez abaixo de 15,0 uC e 5,0 uT, respectivamente, considerados inferiores aos da legislação vigente à época (BRASIL, 2011).

No entanto, destaca-se que apesar da significante quantidade de pesquisas direcionadas ao tratamento de água de abastecimento que utilizam compostos à base de tanino, faltam estudos que esclareçam a interação entre os processos de pré-cloração, coagulação e floculação quando esses coagulantes naturais são utilizados. Nesse sentido, a presente investigação teve como objetivo principal avaliar a influência da pré-cloração no processo de clarificação utilizando coagulante à base de tanino em amostras de água superficial.

\section{METODOLOGIA}

\section{Reagentes químicos}

Os reagentes químicos utilizados nas análises foram de grau analítico. O coagulante adotado foi o Tanfloc SG, produzido pela Tanac (2013), fornecido em pó e certificado pela National Sanitation Foundation. Para os ensaios foi preparada solução estoque de tanino de $10 \mathrm{~g} \mathrm{~L}^{-1}$, conforme Siqueira et al. (2018).

O oxidante utilizado na etapa de pré-cloração da amostra foi solução comercial de hipoclorito de sódio ( $\mathrm{NaClO}$ ) com concentração de 12,0\%, fornecida pela ETA Jaime Câmara. Para a correção do $\mathrm{pH}$, quando necessário, foram utilizadas soluções 0,1 $\mathrm{M}$ de ácido sulfúrico $\left(\mathrm{H}_{2} \mathrm{SO}_{4}\right)$ e $0,05 \mathrm{M}$ de hidróxido de sódio $(\mathrm{NaOH})$, ambas preparadas em água destilada.

\section{Métodos analíticos}

A caracterização da água bruta e tratada foi realizada pela análise dos parâmetros cor aparente, turbidez e $\mathrm{pH}$, de acordo com os métodos preconizados no Standard Methods (APHA, AWWA \& WEF, 2017). A turbidez das amostras foi analisada utilizando-se o turbidímetro de bancada (HACH 2100Q). O espectrofotômetro UV-VIS (HACH, DR 5000) foi empregado para a quantificação da cor aparente $(465 \mathrm{~nm})$. A medição do $\mathrm{pH}$ foi realizada com o pHmêtro de bancada (Tecnal, R-TEC-7-MP). A determinação de cloro residual livre foi feita por meio do método N,N-dietil-p-fenilenodiamina (DPD) colorimétrico.

\section{Coleta e caracterização da água bruta}

A coleta de água bruta foi realizada durante o período de estiagem na entrada da ETA Jaime Câmara, que é abastecida pelo ribeirão João Leite. Foram coletados $100 \mathrm{~L}$ de água, que ficaram armazenados em frascos de polietileno à temperatura ambiente de $24 \pm 2{ }^{\circ} \mathrm{C}$. Antes da coleta, o sistema de pré-cloração foi desativado e a ausência de cloro residual foi confirmada por meio do teste DPD colorimétrico. Previamente aos ensaios de clarificação, a amostra de água bruta foi homogeneizada manualmente e foram analisados os parâmetros cor aparente, $\mathrm{pH}$ e turbidez.

\section{Ensaios de clarificação da água}

Para avaliar o efeito da pré-cloração na clarificação da água utilizando coagulante à base de tanino, foram realizados ensaios em nível de bancada em equipamento jar test (Policontrol, Floc Control II), contendo seis jarros com capacidade volumétrica de $2 \mathrm{~L}$ cada.

As concentrações de cloro residual livre $\left(\mathrm{HClO}_{(\mathrm{NaClO})}\right)$ utilizadas nos ensaios foram 0,8 e $5,0 \mathrm{mg} \mathrm{L}^{-1}$, preparadas com solução de $\mathrm{NaClO}$ a $12 \%$. A concentração de $0,8 \mathrm{mg} \mathrm{L}^{-1}$ corresponde à dosagem utilizada pela ETA Jaime Câmara e a concentração de $5,0 \mathrm{mg} \mathrm{L}^{-1}$ foi adotada em função de ensaios preliminares de cloração da água bruta na ausência de tanino, que resultou em concentração média de $\mathrm{HClO}_{(\mathrm{NaClO})}$ de 2,0 $\mathrm{mg} \mathrm{L}^{-1}$ após 30 min de tempo de contato.

Também foi avaliado o efeito da adição do coagulante com relação à etapa da pré-cloração. Na primeira condição, o tanino e a solução de $\mathrm{NaClO}$ foram adicionados de forma simultânea na água e, em seguida, os ensaios foram repetidos com a adição do tanino após 30 min da etapa de pré-cloração.

Paralelamente, foram realizados ensaios de cloração da água bruta na ausência de tanino e nas mesmas condições de estudo para avaliar o consumo de cloro livre por compostos naturalmente presentes na água. Esses ensaios foram considerados os brancos do estudo. Dessa forma, totalizaram-se cinco condições de ensaios de clarificação da água, conforme apresentado na Figura 1.

O intervalo de pH utilizado em todos os ensaios foi de 6,0 a 8,5 (DI BERNARDO, DANTAS \& VOLTAN, 2017), variando em 0,5 no intervalo. Com base em ensaios preliminares de coagulação, floculação e sedimentação (resultados não apresentados) foram definidos os intervalos da concentração de tanino em função da concentração de $\mathrm{HClO}_{(\mathrm{NaClO})}$ adotada. No ensaio 1, com ausência de $\mathrm{HClO}_{(\mathrm{NaClO})}$ utilizou-se intervalo entre 2,5 e 40,0 $\mathrm{mg} \mathrm{L}^{-1}$ de coagulante tanino. Nos ensaios 2 e 3, com aplicação de $0,8 \mathrm{mg} \mathrm{L}^{-1} \mathrm{de}_{\mathrm{HClO}_{(\mathrm{NaClO})}}$ o intervalo variou de 2,5 a 15,0 $\mathrm{mg} \mathrm{L}^{-1}$. Por fim, nos ensaios 4 e 5 , com adição de $5,0 \mathrm{mg} \mathrm{L}^{-1}$ de $\mathrm{HClO}_{(\mathrm{NaClO})}$ na pré-cloração, o intervalo foi de 2,5 a $45,0 \mathrm{mg} \mathrm{L}^{-1}$. As concentrações de tanino testadas variaram em $2,5 \mathrm{mg} \mathrm{L}^{-1}$ no intervalo adotado. 
As condições adotadas no jar test foram: a) mistura rápida com gradiente de velocidade de $1.000 \mathrm{~s}^{-1}$ e tempo de coagulação de $20 \mathrm{~s}$; b) mistura lenta com gradiente de velocidade de $30 \mathrm{~s}^{-1}$ e tempo de floculação de $20 \mathrm{~min}$; c) tempo de sedimentação de 2,5 min (DI BERNARDO, DANTAS \& VOLTAN, 2017). Esses ensaios foram conduzidos em temperatura ambiente $\left(24 \pm 2^{\circ} \mathrm{C}\right)$.

Nos ensaios 3 e 5, nos quais o tanino foi adicionado após a pré-cloração, primeiramente a água bruta foi mantida em contato com a solução de $\mathrm{NaClO}$ por $30 \mathrm{~min}$ sob agitação constante de $180 \mathrm{~s}^{-1} \mathrm{e}$, em seguida, o tanino foi adicionado. $\mathrm{O}$ ensaio prosseguiu sob as condições operacionais do jar test para as etapas de misturas rápida e lenta e sedimentação supracitadas.

Para cada condição testada foi determinada a dosagem ótima de coagulante e o pH de coagulação, variando de 6,0 a 8,5. Nos ensaios para a determinação da dosagem ótima de coagulante foi utilizado o $\mathrm{pH}$ natural da água, enquanto o pH de coagulação foi obtido usando a dosagem ótima de coagulante. Para a análise dos resultados, as eficiências de remoção de turbidez e cor aparente, em função da dosagem de coagulante ou do $\mathrm{pH}$ de coagulação, foram plotadas no programa SURFER ${ }^{\circledR} 2013$ para a geração de gráficos policromáticos 2D.

\section{RESULTADOS E DISCUSSÃO}

\section{Caracterização da água bruta}

Antes de cada batelada de ensaios, foram aferidos os parâmetros turbidez, cor aparente e $\mathrm{pH}$ das amostras utilizadas de água. Os valores obtidos apresentaram-se nos intervalos entre 12,35 e 15,9 NTU, 78 e $112 \mathrm{uH}$ e pH de 6,8 a 7,2.

\section{Clarificação da água com tanino sem adição de hipoclorito de sódio}

Nas Figuras 2A e 2B estão apresentados os diagramas de remoção de cor aparente e turbidez em função da concentração de tanino e do $\mathrm{pH}$ de coagulação para a concentração ótima de tanino, respectivamente.
Pode-se observar na Figura 2A que o aumento na concentração de tanino de $2,5 \mathrm{mg} \mathrm{L}^{-1}$ em diante ocasionou diminuição na eficiência de remoção de cor aparente. Ainda assim, foi possível obter eficiências de remoção maiores que $90 \%$ para as dosagens entre 2,5 e 5,0 $\mathrm{mg} \mathrm{L}^{-1}$. De 7,5 $\mathrm{mg} \mathrm{L}^{-1}$ de tanino em diante, a eficiência diminui para valores menores que $80 \%$. Para a remoção de turbidez, foi possível obter eficiências de remoção superiores a 90\% para concentração de tanino entre 2,5 e 25,0 $\mathrm{mg} \mathrm{L}^{-1}$. Essa eficiência de remoção de turbidez foi igualmente verificada por Hameed et al. (2016) ao tratarem esgoto doméstico com turbidez considerada baixa, entre 47,0 e 76,0 NTU. Os autores obtiveram eficiência de remoção entre 90 e 95\%, com dosagem de 35,0 $\mathrm{mg} \mathrm{L}^{-1}$ de tanino.

Outros autores verificaram eficiências de remoção de turbidez semelhantes às deste trabalho, no entanto, usando dosagens maiores que a mínima utilizada aqui (2,5 $\mathrm{mg} \mathrm{L}^{-1}$ ). Bongiovani et al. (2016) obtiveram dosagem de tanino entre 5,0 e 17,5 $\mathrm{mg} \mathrm{L}^{-1}$ para amostras de água bruta do Rio Pirapó (Maringá, PR), com turbidez de 56,4 \pm 5,6 NTU e tempo de sedimentação de $20 \mathrm{~min}$. Arantes et al. (2014), ao utilizarem o tanino como auxiliadores da filtração para amostras de água bruta com turbidez de 101,0 a 110,0 uT (água sintética produzida com bentonita), obtiveram dosagens entre 6,0 e 12,0 $\mathrm{mg} \mathrm{L}^{-1}$ para o tempo de sedimentação de $30 \mathrm{~min}$.

Dessa forma, para o tratamento da água em questão, optou-se por adotar a concentração de tanino de $2,5 \mathrm{mg} \mathrm{L}^{-1}$. Os resultados obtidos foram 99,7\% de remoção de cor aparente e 97,5\% de remoção de turbidez. Essa condição foi utilizada para realizar os ensaios para a determinação do pH de coagulação (Figura 2B).

Com relação ao pH de coagulação, foram obtidas eficiências de remoção superiores a 90,0\% para ambos os parâmetros, independentemente do $\mathrm{pH}$ adotado. $\mathrm{O}$ pH de 6,5 apresentou a melhor condição de tratamento, com eficiência de remoção de $96,4 \%$ para cor aparente e $98,5 \%$ para a turbidez, sendo considerado, portanto, o pH de coagulação. Esses resultados assemelham-se ao apresentado por Skoronski et al. (2014), para quem a faixa ideal de coagulação com tanino está entre 4,0 e 8,0. Assim, o tratamento foi realizado no $\mathrm{pH}$ natural da água, evitando o consumo de reagentes para a correção do $\mathrm{pH}$ da água na etapa de clarificação.

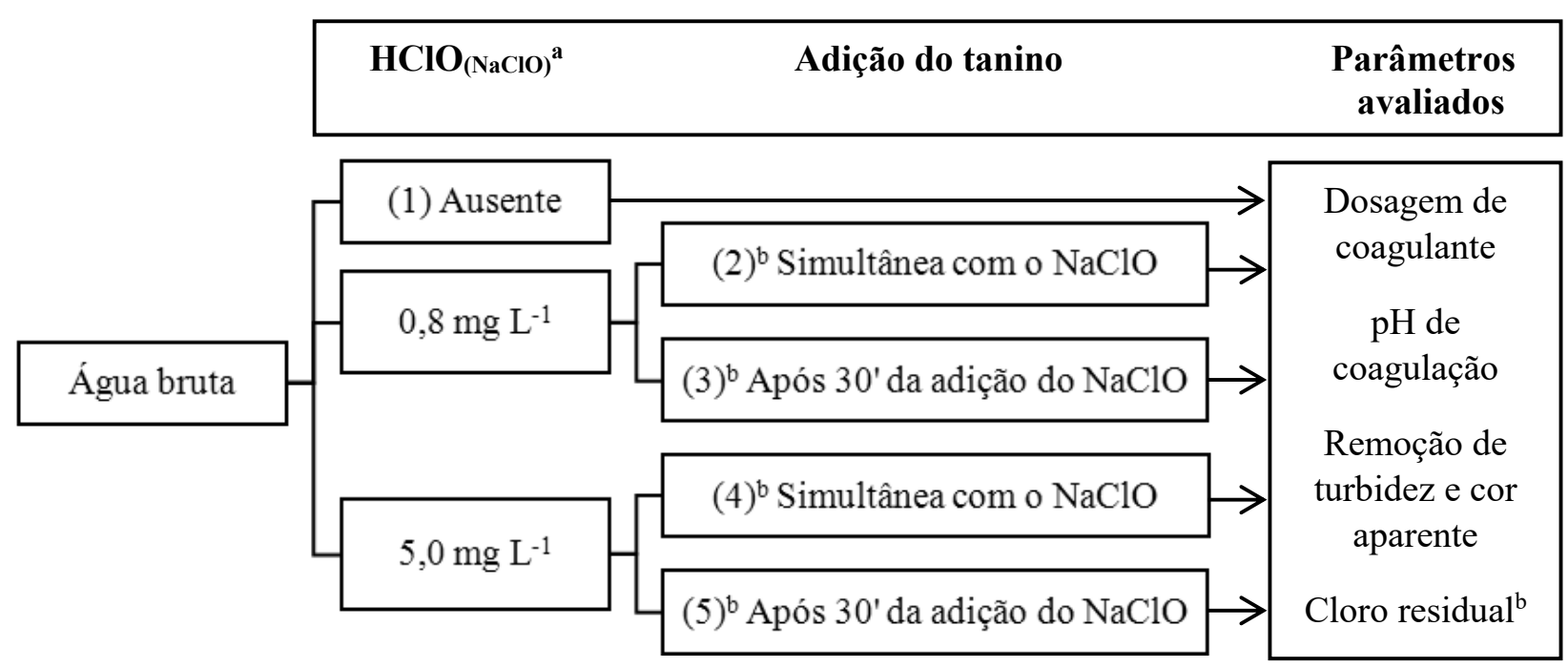

${ }^{a} \mathrm{~A}$ concentração de $\mathrm{HClO}_{\text {(aco) }}$ foi obtida por meio de diluição de solução de $\mathrm{NaClO}$ a 12,0\%.

bEste parâmetro foi analisado nas condições de tratamento com $\mathrm{NaClO}$.

Fonte: elaborada pelos autores.

Figura 1 - Condições dos ensaios de clarificação da água. 


\section{Clarificação da água com tanino adicionado juntamente com hipoclorito de sódio}

Nas Figuras 3A e 3B estão apresentados os diagramas de remoção de turbidez e cor aparente, respectivamente, em função da concentração de tanino, obtidos com base em ensaios de clarificação na presença de 0,8 e 5,0 $\mathrm{mg} \mathrm{L}^{-1} \mathrm{de}$ $\mathrm{HClO}_{(\mathrm{NaClO})}$ e pH natural da água.

Ao analisar ambos os resultados, pode-se verificar que a concentração de $5,0 \mathrm{mg} \mathrm{L}^{-1}$ de $\mathrm{HClO}_{(\mathrm{NaClO})}$, quando comparada com a de $0,8 \mathrm{mg} \mathrm{L}^{-1}$, além de proporcionar menores
A

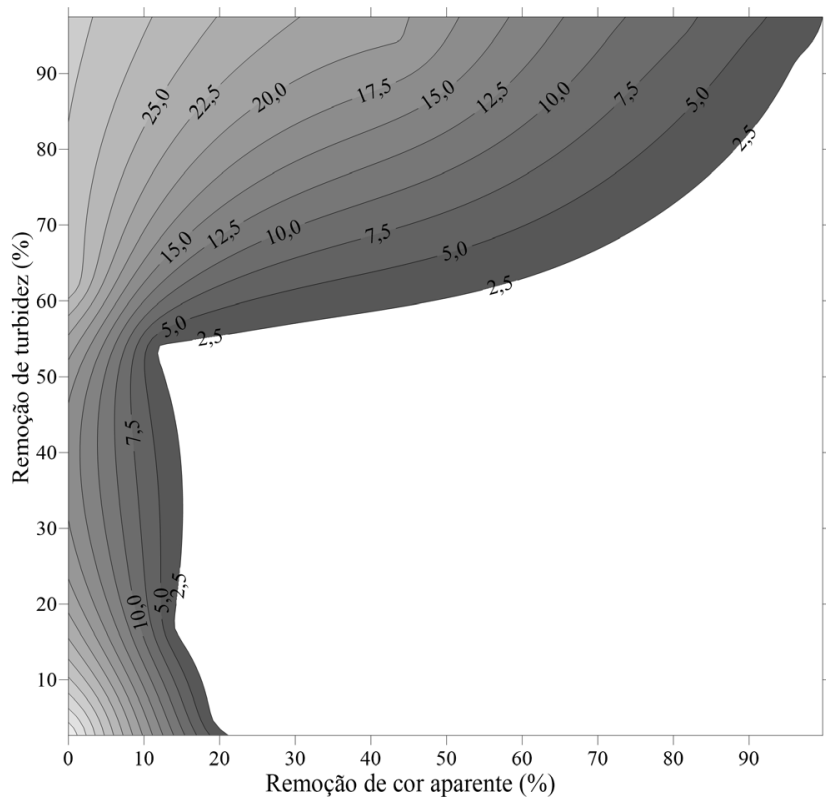

B

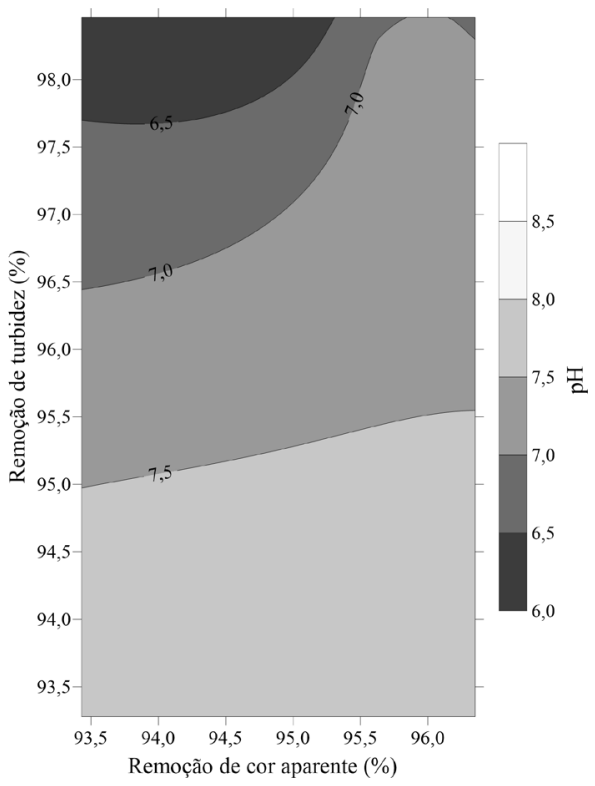

Fonte: elaborada pelos autores.

Figura 2 - Diagramas de remoção de cor aparente e turbidez em função: (A) da concentração de tanino; e (B) do pH de coagulação para a dosagem ótima de tanino.

A

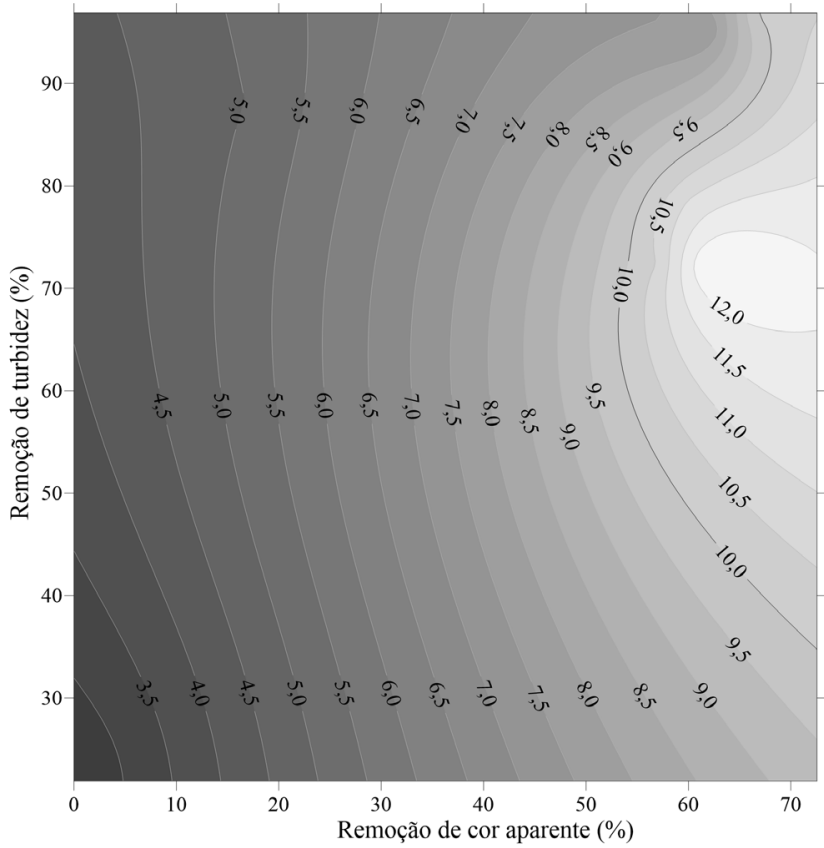

\section{B}

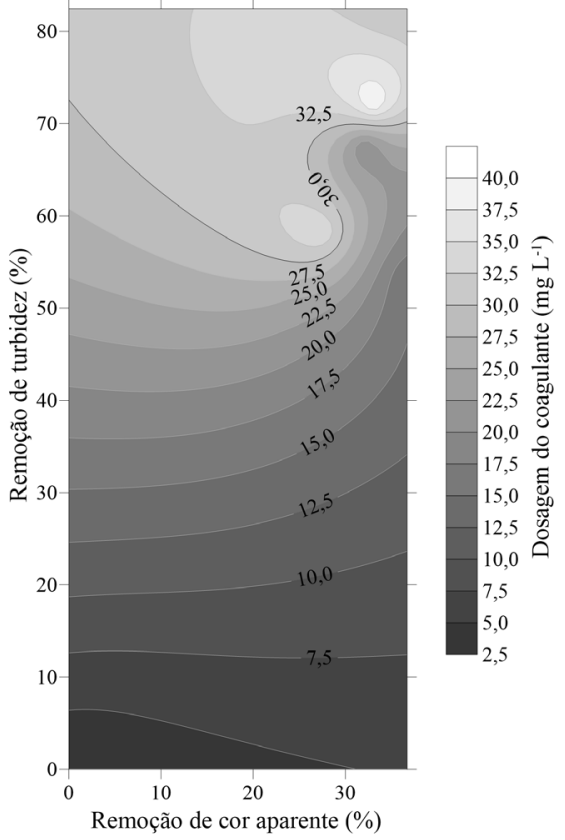

Fonte: elaborada pelos autores

Figura 3 - Diagramas de remoção de turbidez e de cor aparente em função da dosagem de tanino, aplicado simultaneamente ao NaClO, para a obtenção de (A) 0,8 mg L`-1 de cloro residual livre e (B) 5,0 $\mathrm{mg} \mathrm{L}^{-1}$ de cloro residual livre. 
remoções de cor aparente e turbidez, requereu maiores concentrações de tanino no tratamento. A utilização de $0,8 \mathrm{mg} \mathrm{L}^{-1}$ de $\mathrm{HClO}_{(\mathrm{NaClO})}$ proporcionou remoção de turbidez na ordem de $90 \%$ para dosagem de tanino entre 4,5 e $10,5 \mathrm{mg} \mathrm{L}^{-1}$, ao passo que, para a remoção de 70\% de cor aparente, esse intervalo ficou entre 9,0 e 11,0 $\mathrm{mg} \mathrm{L}^{-1}$.

Dosagens maiores que os valores apresentados causaram diminuição na eficiência de remoção para ambos os parâmetros. Essa situação pode ser explicada considerando-se que a remoção de turbidez por floculação, ao utilizar polímeros cationicos, ocorre por meio de dois mecanismos principais: o primeiro relacionado à neutralização de cargas em virtude da interação eletrostática entre a partícula que apresenta, geralmente, superfície negativamente carregada e as cargas positivas do polímero catiônico; e outro baseado na formação de pontes, quando segmentos de polímeros adsorvidos sobre as partículas estendem-se em solução e atam-se a outras partículas, unindo-as (BOLTO \& GREGORY, 2007).

Hameed et al. (2016) explicam que, para a ocorrência do mecanismo de floculação por ponte, é preciso que parte da superfície das partículas esteja desocupada, de forma que os segmentos das cadeias de polímeros possam se ligar às cargas da partícula não neutralizadas. Consequentemente, o excesso de polímero catiônico pode cobrir totalmente a superfície das partículas (pela neutralização de carga), impedindo o desenvolvimento do mecanismo de floculação por ponte e diminuindo a eficiência de remoção de turbidez. Essa situação teria refletido na remoção da cor aparente, visto que a turbidez é considerada um interferente desse parâmetro.

Com relação ao resultado das análises de consumo de $\mathrm{HClO}_{(\mathrm{NaClO})}$, tanto na amostra com tanino quanto no branco (amostra de água bruta sem tanino), os valores obtidos estavam abaixo do limite de detecção do método $\left(0,1 \mathrm{mg} \mathrm{L}^{-1}\right)$.

Quando a concentração de $\mathrm{HClO}_{(\mathrm{NaClO})}$ foi aumentada para 5,0 $\mathrm{mg} \mathrm{L}^{-1}$, a máxima remoção de turbidez foi de $80 \%$ para a dosagem de tanino superior a $30,0 \mathrm{mg} \mathrm{L}^{-1}$ e de $40 \%$ de cor aparente obtida com dosagem de 7,5 $\mathrm{mg} \mathrm{L}^{-1}$ ou mais. A maior demanda de tanino para a remoção de turbidez nesses ensaios pode estar associada à possível oxidação do coagulante pelo $\mathrm{HClO}_{(\mathrm{NaClO})}$. Tal hipótese sustenta-se nos resultados das análises de $\mathrm{HClO}_{(\mathrm{NaClO})}$ realizadas em amostras tratadas com tanino (valores abaixo do limite de detecção, de $0,1 \mathrm{mg} \mathrm{L}^{-1}$ ) e nos brancos $\left(1,1 \mathrm{mg} \mathrm{L}^{-1}\right.$ de $\left.\mathrm{HClO}_{(\mathrm{NaClO})}\right)$ no fim dos ensaios.

Após a análise dos resultados, determinou-se para a tratabilidade da água pré-oxidada com 0,8 e 5,0 $\mathrm{mg} \mathrm{L}^{-1}$ de $\mathrm{HClO}_{(\mathrm{NaClO})}$ as dosagens de tanino de 10,0 e 30,0 $\mathrm{mg} \mathrm{L}^{-1}$, respectivamente. Essas dosagens foram utilizadas para determinar o pH de coagulação. No que diz respeito ao $\mathrm{pH}$ ótimo de coagulação para a clarificação da água, na presença de 0,8 e 5,0 $\mathrm{mg} \mathrm{L}^{-1}$ de $\mathrm{HClO}_{(\mathrm{NaClO})}$ ocorreram remoções abaixo de $80 \%$ de turbidez e inferiores a $70 \%$ de cor aparente, no intervalo de $\mathrm{pH}$ entre 7,0 e 8,0. Esses resultados são corroborados por Skoronski et al. (2014), que consideraram a faixa ideal de coagulação com tanino entre 4,0 e 8,0.

Nos ensaios com pH inferiores a 7,0, na presença de $0,8 \mathrm{mg} \mathrm{L}^{-1} \mathrm{de}_{\mathrm{HClO}}{ }_{(\mathrm{NaClO})}$, observou-se que as eficiências de remoção desses parâmetros foram de 71,6\% para turbidez e de $62,7 \%$ para cor aparente. Em contrapartida, na presença de $5,0 \mathrm{mg} \mathrm{L}^{-1}$ de $\mathrm{HClO}_{(\mathrm{NaClO})}$ foi verificada remoção de turbidez de $37,1 \%$, porém não foi observada remoção de cor aparente.

Acredita-se que essa situação esteja associada com a influência do pH nas formas predominantes de cloro residual e não na atuação do tanino como coagulante. De acordo com Souza et al. (2018), a espécie de maior poder oxidante, o $\mathrm{HClO}$ (ácido hipocloroso), é predominante até o pH igual a 7,0, enquanto a forma $\mathrm{OCl}^{-}$(íon hipoclorito) de menor poder oxidante predomina em $\mathrm{pH}$ mais básicos. Sendo assim, em pH inferiores a 6,5, ao prevalecerem as formas oxidantes do cloro residual, teve-se maior oxidação do tanino, principalmente nas maiores dosagens, o que comprometeu o processo de tratamento.

\section{Clarificação da água com adição do tanino após 30 min da etapa de pré-cloração com hipoclorito de sódio}

Dada a influência do $\mathrm{NaClO}$ no processo de tratamento da água por coagulação, resultando no aumento de agente coagulante, optou-se por realizar ensaios nos quais o oxidante foi adicionado separadamente do tanino, neste caso, $30 \mathrm{~min}$ antes. Nas Figura 4A e 4B estão apresentados os diagramas de remoção de cor aparente e de turbidez em função das dosagens de tanino para água pré-clorada com $0,8 \mathrm{e}$ $5,0 \mathrm{mg} \mathrm{L}^{-1} \mathrm{de} \mathrm{HClO}_{(\mathrm{NaClO})}$, respectivamente $\mathrm{O} \mathrm{pH}$ do ensaio foi o natural da água.

Pode-se observar na Figura $4 \mathrm{~A}$ que a remoção de turbidez alcançou valores superiores a 70\% com dosagem de tanino entre 6,5 e 15,0 $\mathrm{mg} \mathrm{L}^{-1} \mathrm{e}$, para cor aparente, remoção de $80 \%$ com dosagem de tanino entre 4,5 e 6,0 $\mathrm{mg} \mathrm{L}^{-1}$.

No ensaio com adição de 5,0 $\mathrm{mg} \mathrm{L}^{-1}$ de $\mathrm{HClO}_{(\mathrm{NaClO})}$ (Figura 4B), eficiências de remoção acima de $70 \%$ de turbidez foram alcançadas para dosagens superiores a $12,5 \mathrm{mg} \mathrm{L}^{-1}$, enquanto remoções de $60 \%$ de cor aparente apresentaram dosagens
A

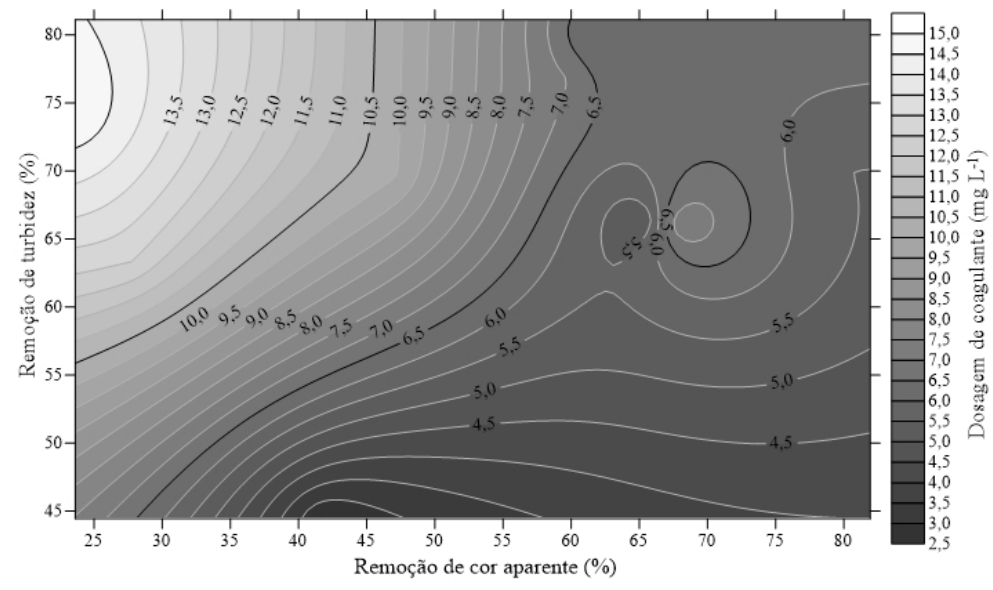

B

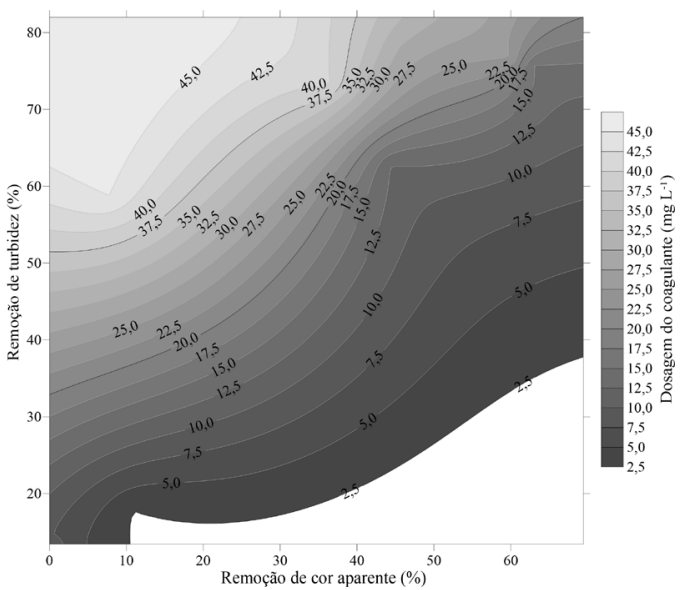

Fonte: elaborada pelos autores

Figura 4 - Diagramas de remoção de turbidez e de cor aparente, em função das dosagens de tanino e cloro residual livre: $(A)$ 0,8 mg $\mathrm{L}^{-1}$ e (B) 5,0 mg $\mathrm{L}^{-1}$ de $\mathrm{HCIO}_{(\mathrm{NaClO})}$ 
abaixo de 22,5 $\mathrm{mg} \mathrm{L}^{-1}$. As menores eficiências de remoção e a elevada dosagem de tanino para remoção de turbidez podem, igualmente, estar associadas à oxidação do coagulante por parte do $\mathrm{HClO}_{(\mathrm{NaClO})}$, assim como verificado no ensaio em que esse composto foi adicionado simultaneamente com o coagulante. Essa hipótese foi levantada porque os resultados da concentração de $\mathrm{HClO}_{(\mathrm{NaClO})}$ foram semelhantes.

Nesses ensaios, também foi possível verificar tendência de diminuição na eficiência de remoção de cor aparente com o aumento da dosagem de tanino de 22,5 $\mathrm{mg} \mathrm{L}^{-1} \mathrm{em}$ diante. Isso também foi observado por Silveira et al. (2019); quando esse coagulante é utilizado em altas dosagens, pode conferir cor mais elevada em virtude da cor natural do tanino. Dessa forma, considerando-se a remoção de ambos os parâmetros, as dosagens ótimas de tanino para a clarificação da água em estudo com adição de 0,8 e 5,0 $\mathrm{mg} \mathrm{L}^{-1}$ de $\mathrm{HClO}_{(\mathrm{NaClO})}$ foram, respectivamente, 6,5 e 20,0 $\mathrm{mg} \mathrm{L}^{-1}$.

Com relação à influência do $\mathrm{pH}$, adotando-se as dosagens ótimas de tanino e a adição de $\mathrm{HClO}_{(\mathrm{NaClO})}$ supracitadas, chegou-se aos resultados apresentados nas Figuras 5A e 5B. Verifica-se que remoções superiores a $60 \%$ de cor aparente e turbidez foram alcançadas para valores de $\mathrm{pH}$ de 7,0 e 7,5, para ambas as concentrações de $\mathrm{HClO}_{(\mathrm{NaClO})}$ adotadas. Tais resultados corroboram a faixa ideal de coagulação da água com tanino entre 4,0 e 8,0 (SKORONSKI et al., 2014).

\section{Comparação entre os ensaios de clarificação que utilizaram tanino puro, tanino adicionado simultaneamente e após 30 min da adição de hipoclorito de sódio}

Na Figura 6 são apresentadas as eficiências de remoção de turbidez (principal parâmetro de eficiência do processo de clarificação) obtidas nos ensaios em que o tanino foi adicionado juntamente com o $\mathrm{NaClO}$ e após $30 \mathrm{~min}$ deste. Os valores de $\mathrm{pH}$ e de concentração do coagulante tanino foram aqueles considerados como as condições ótimas de tratamento.

Pode ser observado que a concentração de $0,8 \mathrm{mg} \mathrm{L}^{-1}$ de $\mathrm{HClO}_{(\mathrm{NaClO})}$ independentemente de se o oxidante foi adicionado junto com o tanino ou $30 \mathrm{~min}$ antes, proporcionou tanto as menores dosagens de coagulante quanto as maiores eficiências na remoção de turbidez, quando comparada com os resultados obti-

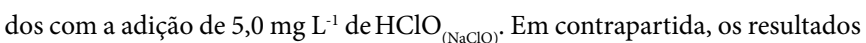

da clarificação da água sem a etapa de pré-oxidação resultaram na concentração de 2,5 $\mathrm{mg} \mathrm{L}^{-1}$ de tanino, com remoção de 97,5\% de turbidez.

Alam et al. (2008) utilizaram dióxido de cloro $\left(0,6\right.$ a $\left.1,0 \mathrm{mg} \mathrm{L}^{-1}\right)$ na pré-oxidação de água destinada ao consumo humano e não obtiveram melhora significativa na qualidade da água. Xie et al. (2016) indicam que a pré-oxidação com compostos clorados pode ser limitada quando aplicada em águas superficiais contendo pouca ou nenhuma alga, situação da água utilizada no presente estudo em vista dos baixos valores de turbidez obtidos.

Outro fato a se destacar são as maiores dosagens de tanino utilizadas quando os ensaios foram realizados com a concentração de $5,0 \mathrm{mg} \mathrm{L}^{-1}$ de $\mathrm{HClO}_{(\mathrm{NaClO})}$ que, ainda assim, resultaram em menores eficiências de remoção de turbidez.

Ma et al. (2012) avaliaram o efeito da pré-cloração na remoção de algas por coagulação com alumina (óxido de alumínio). Esses autores verificaram que doses de cloro entre 0,0 e 4,0 $\mathrm{mg} \mathrm{L}^{-1}$ afetaram as tendências das curvas resposta da dosagem de alumina quanto à remoção das algas, o que indica que maiores concentrações de cloro necessitaram de maiores dosagens de alumina, sem, no entanto, obter remoção proporcional. Por exemplo, para a aplicação de concentrações inferiores a $2,0 \mathrm{mg} \mathrm{L}^{-1}$ de cloro foi preciso variar de 2,0 para $2,7 \mathrm{mg} \mathrm{L}^{-1}$ de alumina para se obterem $38,0 \%$ a mais na remoção de algas. Já ao se utilizar entre 2,5 e $3,5 \mathrm{mg} \mathrm{L}^{-1}$ de cloro e aumentar a dosagem de coagulante de 2,0 para $2,4 \mathrm{mg} \mathrm{L}^{-1}$ o aumento na remoção na remoção de algas foi de $2,1 \%$. Tais resultados foram atribuídos à constituição do material orgânico dissolvido no meio.

Xie et al. (2013) explicaram que oxidantes fortes propiciam a formação de material orgânico com baixo peso molecular, que são mais dificilmente removidos por coagulação e floculação. Essa condição pode ser intensificada com o tipo e concentração do oxidante.

Além disso, neste estudo também se levanta a possibilidade de a elevada concentração de $\mathrm{HClO}_{(\mathrm{NaClO})}$ ter oxidado parte do coagulante tanino, tendo em vista os resultados da análise de cloro residual obtidos em amostras tratadas e nos brancos, conforme explicado anteriormente. Libânio (2016) explica que o cloro pode ser consumido pela oxidação de compostos nitrogenados, matéria orgânica e inorgânica.
A

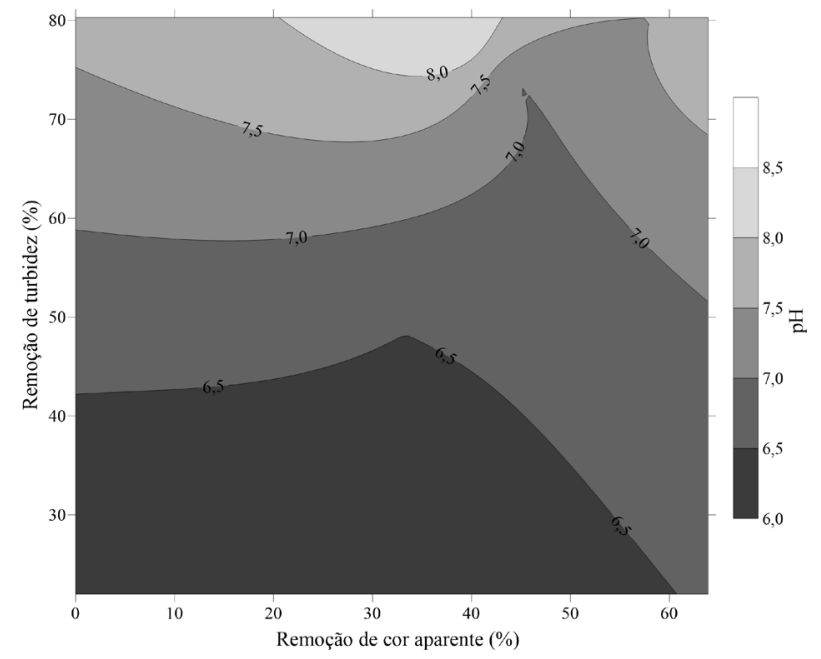

B

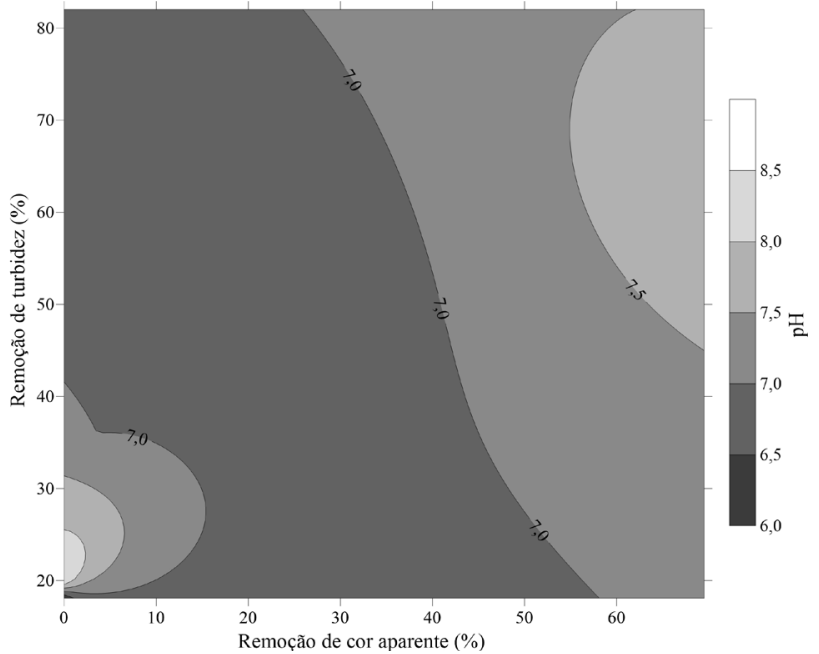

Fonte: elaborada pelos autores.

Figura 5 - Diagramas de remoção de turbidez e de cor aparente em função do pH na presença de cloro residual livre: (A) 0,8 mg L-1 e (B) 5,0 mg L-1. 


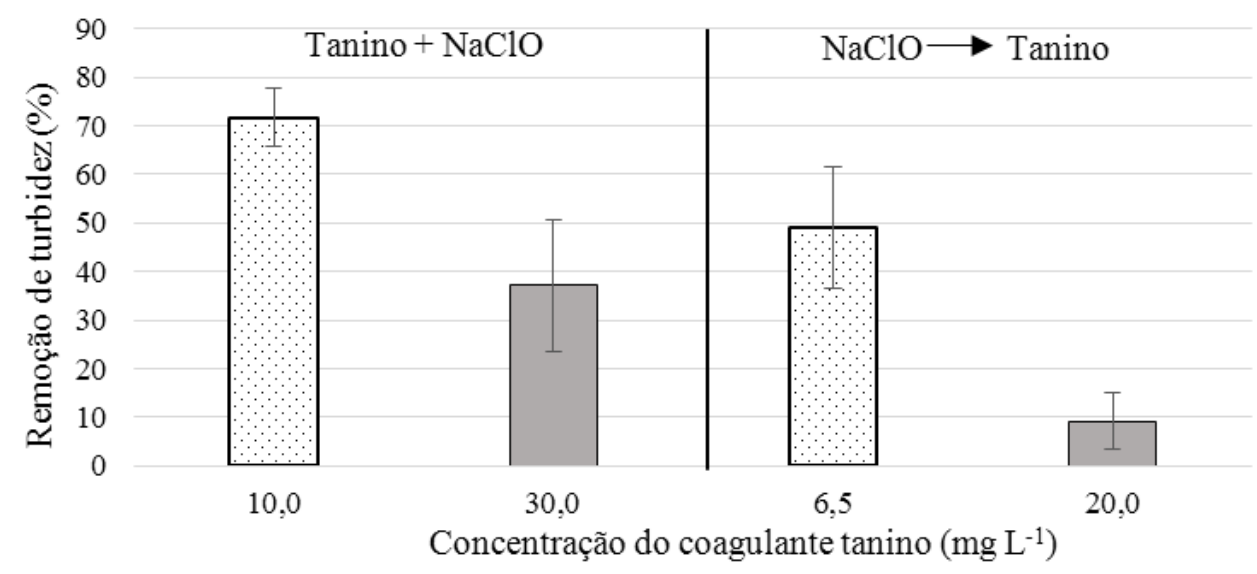

$$
0,8 \mathrm{mg} \mathrm{L}^{-1} \mathrm{HClO}_{(\mathrm{NaClO})} \quad \square 5,0 \mathrm{mg} \mathrm{L}^{-1} \mathrm{HClO}_{(\mathrm{NaClO})}
$$

$\mathrm{NaClO}$ : hipoclorito de sódio; $\mathrm{HClO}_{\text {(Naclo) }}$ : cloro residual livre.

Fonte: elaborada pelos autores.

Figura 6 - Eficiências de remoção de turbidez nos ensaios com adição simultânea de tanino e cloro residual livre(tanino + hipoclorito de sódio) e após 30 min da adição do cloro residual livre (hipoclorito de sódio $\rightarrow$ Tanino).

Quanto à remoção de cor aparente, nos ensaios conduzidos na presença de $0,8 \mathrm{mg} \mathrm{L}^{-1}$ de $\mathrm{HClO}_{(\mathrm{NaClO})}$, considerando-se as mesmas condições de $\mathrm{pH}$ e a dosagem de coagulante na remoção de turbidez, as eficiências de remoção foram 62,7 e 15,6\% para adição simultânea e separada do oxidante, respectivamente. No entanto, quando se utilizaram $5,0 \mathrm{mg} \mathrm{L}^{-1} \mathrm{de}_{\mathrm{HClO}_{(\mathrm{NaClO})}}$ não foi constatada remoção desse parâmetro, para ambas as condições. Esses resultados podem ter sido influenciados pela turbidez remanescente na amostra, que foi consideravelmente menor para essa condição.

\section{CONCLUSÕES}

Dos resultados obtidos no presente estudo, pode-se concluir que:

- para as características da água bruta e os parâmetros operacionais utilizados no ensaio de clarificação da água somente com o coagulante (ensaio 1), a dosagem ótima de tanino foi de $2,5 \mathrm{mg} \mathrm{L}^{-1}$, resultando em $99,7 \%$ de remoção de cor aparente e 97,5\% de remoção de turbidez;

- com a adição da etapa de pré-oxidação, obtiveram-se os melhores resultados para adição simultânea do oxidante $\mathrm{NaClO}$ e do coagulante tanino (ensaio 2) nas dosagens de $0,8 \mathrm{mg} \mathrm{L}^{-1}$ de $\mathrm{HClO}_{(\mathrm{NaClO})}$ e $10,0 \mathrm{mg} \mathrm{L}^{-1}$ de tanino, com eficiências de remoção de turbidez de 71,6\% e de cor aparente de 62,7\%;

- a pré-cloração afetou negativamente a eficiência do tratamento da água com o coagulante tanino;
- quanto maior a concentração de $\mathrm{HClO}_{(\mathrm{NaClO})}$ maior a dosagem de tanino requerida para a clarificação da água, resultando em menores eficiências de remoção de turbidez e cor aparente;

- a adição do coagulante, após $30 \mathrm{~min}$ da adição do $\mathrm{NaClO}$, apesar de ter proporcionado menores dosagens de tanino para a condição ótima de coagulação, afetou negativamente as eficiências de remoção de turbidez e cor aparente;

- o pH de coagulação, com o uso de tanino, não sofreu alterações significativas com a adição do $\mathrm{NaClO}$. Porém, essa variável tem influência nas formas predominantes do $\mathrm{HClO}$ e, por consequência, na etapa de coagulação.

\section{AGRADECIMENTOS}

Agradecimentos à Coordenação de Aperfeiçoamento de Pessoal de Nível Superior (CAPES), a concessão de bolsas de estudos de mestrado ao Programa de PósGraduação em Engenharia Ambiental e Sanitária da Universidade Federal de Goiás.

\section{CONTRIBUIÇÃO DOS AUTORES}

Silveira, J.R.: Conceituação, Curadoria de Dados, Análise Formal, Metodologia, Escrita, Recursos - Primeira Redação, Revisão e Edição. Souza, V.M. de Conceituação, Curadoria de Dados, Metodologia, Escrita, Recursos — Primeira Redação. Cuba, R.M.F.: Administração do projeto, Conceituação, Supervisão, Escrita - Revisão e Edição. Teran, F.J.C.: Supervisão - Revisão.

\section{REFERÊNCIAS}

ALAM, M. Z. B.: CANTWELL, R. E.: HOFMANN, R.: ANDREWS, R. C.: RAND, J. L.; GAGNON, G. A.; VANDERMARCK, M.; MOFFAT, E.; ANDREWS, S. A. Effect of $\mathrm{ClO}_{2}$ pretreatment on subsequent water treatment processes. Journal of Environmental Engineering, v. 134, n. 6, p. 478-485, 2008. https://doi. org/10.1061/(asce)0733-9372(2008)134:6(478)
AMERICAN PUBLIC HEALTH ASSOCIATION (APHA); AMERICAN WATER WORKS ASSOCIATION (AWWA); WATER ENVIRONMENT FEDERATION (WEF). Standard methods for the examination of water and wastewater. 23a ed. Washington: American Public Health Association, 2017. 
ARANTES, C. C.; RIBEIRO, T. A. P.; PATERNIANI, J. E. S.; TATEOKA, M. S. S.; SILVA, G. K. Uso de coagulantes naturais à base de moringa oleifera e tanino como auxiliares da filtração em geotêxtil sintético não tecido. Engenharia Agrícola, v. 34, n. 4, p. 780-788, 2014. https://doi.org/10.1590/ S0100-69162014000400017

BARTIKO, D; JULIO, M. Construção e emprego de diagramas de coagulação como ferramenta para o monitoramento contínuo da floculação em águas de abastecimento. Revista Ambiente \& Água - An Interdisciplinary Journal of Applied Science, v. 10, n. 1, 2015. https://doi.org/10.4136/ambi-agua.1239

BELTRÁN-HEREDIA, J.; SANCHEZ-MARTÍN, J.; GÓMEZ-MUÑOZ, M. C. New coagulant agents from tannin extracts: Preliminary optimisation studies. Chemical Engineering Journal, v. 162, n. 3, p. 1019-1025, 2010. https://doi. org/10.1016/j.cej.2010.07.011

BOLTO, B.; GREGORY, J. Organic polyelectrolytes in water treatment. Water Research, v. 41, n. 11, p. 2301-2324, 2007. https://doi.org/10.1016/j. watres.2007.03.012

BONGIOVANI, M. C.; KONRADTMORAES, L. C.; BERGAMASCO, R.; LOURENÇO, B. S. S.; TAVARES, C. R. G. Os benefícios da utilização de coagulantes naturais para a obtenção de água potável. Acta Scientiarum. Technology, v. 32, n. 2, p. 167-170, 2010. https://doi.org/10.402/actascitechnol. v32i2.8238

BRASIL. Portaria de Consolidação no 5, de 28 de setembro de 2017. Ministério da Saúde. Consolidação das normas sobre as ações e os serviços de saúde do Sistema Único de Saúde. Diário Oficial da União. Brasília: Ministério da Saúde, 2017.

BRASIL. Portaria n 2914, de 12 de dezembro de 2011. Ministério da Saúde. Dispõe sobre os procedimentos de controle e de vigilância da qualidade da água para consumo humano e seu padrão de potabilidade. Diário Oficial da União. Brasília: Ministério da Saúde, 2011.

DI BERNARDO, L.; DANTAS, A. D. B.; VOLTAN, P. E. N. Métodos e Técnicas de Tratamento de Água. 3a ed. São Carlos: LDiBe Editora, 2017. 1296 p.

HAMEED, Y. T.; IDRIS, A.; HUSSAIN, S. A.; ABDULLAH, N. A tannin-based agent for coagulation and flocculation of municipal wastewater: Chemical composition, performance assessment compared to Polyaluminum chloride, and application in a pilot plant. Journal of Environmental Management, v. 184 , p. 494-503, 2016. https://doi.org/10.1016/j. jenvman.2016.10.033

HELLER, L.; PÁDUA, V. L. (Orgs.). Abastecimento de água para consumo humano. $1^{\text {a }}$ ed. Belo Horizonte: UFMG, 2006. 859 p.

LIBÂNIO, M. Fundamentos de qualidade e tratamento de água. $4^{a}$ ed. Campinas: Editora Átomo, 2016. 640 p.

MA, M.; LIU, R.; LIU, H.; QU, J.; JEFFERSON, W. Effects and mechanisms of pre-chlorination on Microcystis aeruginosa removal by alum coagulation: Significance of the released intracellular organic matter. Separation and Purification Technology, v. 86, p. 19-25, 2012. http://doi.org/10.1016/j. seppur.2011.10.015
MANGRICH, A. S.; DOUMER, M. E.; MALLMANN, A. S.; WOLF, C. R. Química verde no tratamento de águas: uso de coagulante derivado de tanino de Acacia mearnsii. Revista Virtual de Química, v. 6, n. 1, p. 2-15, 2014. http:// dx.doi.org/10.5935/1984-6835.20140002

NETO, J. C.; NEYCOMBE, G. Oxidation of cyanobacteria and their metabolites in water treatment facilities: the state of art. Engenharia Sanitária e Ambiental, v. 22, n. 5, p. 829-840, 2017. https://doi.org/10.1590/ S1413-41522017148174

OLADOJA, N. A.; SALIU, T. D.; OLOLADE, I. A.; ANTHONY, E. T.; BELLO, G. A. A new indigenous green option for turbidity removal from aqueos system. Separation and Purification Technology, v. 186, p. 166-174, 2017. https://doi. org/10.1016/j.seppur.2017.05.054

SANTOS, W. N. A.: BATISTA, I. F;: MICHELAN, D. C. G.S.:SANTOS, D. G.; MENDONÇA L. C. Uso do sulfato de alumínio e do tanino vegetal no tratamento de água. Anais do X Simpósio de Engenharia de Produção de Sergipe, p. 384-394, 2018.

SKORONSKI, E.; NIERO, B.; FERNANDES, M.; ALVES, M. V.; TREVISAN, V. Estudo da aplicação de tanino no tratamento de água para abastecimento captada no rio Tubarão, na cidade de Tubarão, SC. Ambiente \& Água, v. 9, n. 4, 2014. https://doi.org/10.4136/ambi-agua.1303

SILVEIRA, T. N.; BARBOSA, M. G. N.; PEQUENO, L. A. B. SANTOS, W. B. FERREIRA, W. B. Performance de coagulantes orgânicos e inorgânicos por meio de diagrama de coagulação em águas naturais. Revista Eletrônica de Gestão e Tecnologias Ambientais, v. 7, n. 7. p. 16-25, 2019. https://doi. org/10.9771/gesta.v7i7.28068

SIQUEIRA, A. P. S.; SILVA, C. N.; REZENDE, L. C. S. H.; MILANI, R. G.; YAMAGUCHI, N. U. Análise da performance dos coagulantes naturais moringa oleifera e tanino como alternativa ao sulfato de alumínio para o tratamento de água. Enciclopédia Biosfera, v. 15, n. 27, p. 18-29, 2018. https:// doi.org/10.18677/encibio_2018a112

SOUZA, B. P.; LIMA, D. R. S.; AQUINO, S. F.; QUARESMA, A. V.; BAÊTA, B. E. L.; LIBÂNIO, M. Oxidação de fármacos por cloro e formação de subprodutos em amostras aquosas em escala de bancada. Engenharia Sanitária e Ambiental, v. 23, n. 2, p. 207-216, 2018. https://doi.org/10.1590/s1413-41522018155335

TANAC. Tanfloc SG. 2013. Disponível em: https://www.tanac.com.br/sites/ default/files/CT_TANFLOC_SG_PT_O.pdf. Acesso em: 24 jul. 2019.

THAKUR, S. S.; CHOUBEY, S. Use of Tannin based natural coagulants for water treatment: An alternative to inorganic chemicals. International Journal of ChemTech Research, v. 6, n. 7, p. 3628-3634, 2014

XIE, P.; MA, J.; FANG, J.; GUAN, Y.; YUE, S.; LI, X.; CHEN, L. Comparison of permanganate preoxidation and preozonation on algae containing water: cell integrity, characteristics, and chlorinated disinfection byproduct formation. Environmental Science \& Technology, v. 47, n. 24, p. 14051-14061, 2013. https://doi.org/10.1021/es4027024

XIE, P.; CHEN, Y.; MA, J.; ZHANG, X.; ZOU, J.; WANG, Z. A mini review of preoxidation to improve coagulation. Chemosphere, v. 155, p. 550-563, 2016. https://doi.org/10.1016/j.chemosphere.2016.04.003 